\title{
La métalangue sémantique naturelle et la lutte contre le syndrome du franchissement du gué
}

\author{
Bert Peeters \\ Macquarie University (Sydney) \\ Bert.Peeters@humn.mq.edu.au
}

\section{Définitions}

«Instrument efficace dans la lutte contre le syndrome du franchissement du gué, la MSN est indispensable à tous ceux qui ont souci de bien se faire comprendre. » C'est par ces mots que je terminais, le 5 juillet 2007, ma conférence plénière au colloque international de Montpellier, consacré aux enjeux de la communication interculturelle (Peeters à paraître a). Au cours de la conférence, j'avais fait valoir que ledit syndrome se trouve être particulièrement répandu parmi les spécialistes de l'interculturel et qu'il est d'autant plus dangereux qu'il reste peu connu, n'en déplaise à celui qui l'a reconnu pour ce qu'il est, à savoir l'inimitable Georges Kleiber, que je cite : «Ce syndrome, relevé pour la première fois au Moyenâge chez les moines de l'Oelenberg (à Reiningue, près de Mulhouse), est bien connu : on continue de sauter d'une pierre à une autre, sans jamais tomber à l'eau, mais on oublie de franchir la rivière ! » (Kleiber 2001: 3).

De quelle façon le syndrome du franchissement du gué affecte-t-il la communauté des spécialistes de l'interculturel ? Il l'affecte en ce que, bien trop souvent, on se propose de décrire et d'expliciter ce qui est culturellement spécifique, en soi ou bien en comparaison avec ce qu'on trouve ailleurs, mais sans arriver à ce qui devrait être l'objectif ultime : une description où tout le monde se retrouve. Je donnais l'exemple $\mathrm{du}$ japonais amae, qui a fait l'objet de multiples descriptions, y compris en anglais et en français, lesquelles donnent au lecteur une idée de ce qu'est l'amae; mais il s'agit d'approximations, au mieux, et de défigurations, au pire, car suite à l'emploi de tournures telles que désir d'indulgence, extrême dépendance, se prévaloir des affections d'un prochain, refus de l'autonomie, fuite devant les contraintes de la réalité, etc., un concept typiquement japonais a été représenté à travers le prisme d'une macroculture occidentale, à travers des lunettes colorées qui filtrent l'objet et le défigurent. Les Japonais euxmêmes, à commencer par Takeo Doi, l'ont décrit aussi, en japonais, et leurs descriptions peuvent être appréciées à leur juste valeur par les Japonais et par ceux qui ont une bonne connaissance de la culture et de la langue japonaises ; mais d'autres en sont pour leurs frais, car pour eux les descriptions en langue japonaise restent impénétrables. Il est vrai que l'ouvrage de Takeo Doi a été traduit en français, mais ce texte (Doi 1982) recourt au même genre de vocabulaire et fournit donc aussi des approximations, au mieux, et des défigurations, au pire. Je faisais remarquer que l'idéal serait une description que les nonJaponais arrivent à comprendre sans la moindre difficulté, et dans laquelle les Japonais eux-mêmes se reconnaissent (ou qu'on peut facilement modifier au cas où ils ne s'y reconnaîtraient pas). J'enchaînais en disant que le moyen de réaliser une description de ce genre existe : le « secret » consiste à se servir de la métalangue sémantique naturelle (désignée ci-dessus et ci-après à l'aide du sigle MSN).

Comme à Chypre, il y a deux ans, lors d'un colloque de lexicographie où je défendais la thèse (Peeters à paraître b) que la MSN permet de jeter une nouvelle lumière sur la question des faux amis, je dirai, à l'instar du maître de philosophie de Molière, à ceux qui m'écoutent ou me lisent : "Vous entendez cela, et vous connaissez la métalangue sémantique naturelle sans doute ?» - et aux Messieurs et Mesdames Jourdain qui me répondront : «Oui, mais faites comme si je ne la connaissais pas. Expliquez-moi ce que cela veut dire $»$, je ferai remarquer qu'il s'agit d'une langue qui, comme toutes les langues naturelles, a un lexique et une grammaire (contrairement à un grand nombre d'autres métalangues). Seulement, ce lexique et cette grammaire sont d'un type particulier : ils permettent à la langue dont ils relèvent d'assumer le rôle de «métalangue » universelle et dès lors culturellement neutre, qui échappe au dogme du traduttore 
traditore, de sorte que la description qu'on offre en anglais, ou en français, puisse être transposée sans difficulté et sans défiguration dans d'autres langues, quelles qu'elles soient, y compris le japonais. La MSN est dès lors un outil descriptif rigoureux qui permet de parler, non seulement des faux amis (comme je l'ai fait à Chypre), mais de tous les mots dans toutes les langues - et non seulement de tous les mots dans toutes les langues, mais aussi d'autres faits linguistiques, qu'il s'agisse de structures grammaticales, de normes communicatives ou de valeurs culturelles, et ainsi de suite (comme je l'ai fait à Montpellier).

Pour le concept japonais de l'amae, j'ai proposé (Peeters à paraître a) l'explicitation ${ }^{1}$ ou formule sémantique suivante :

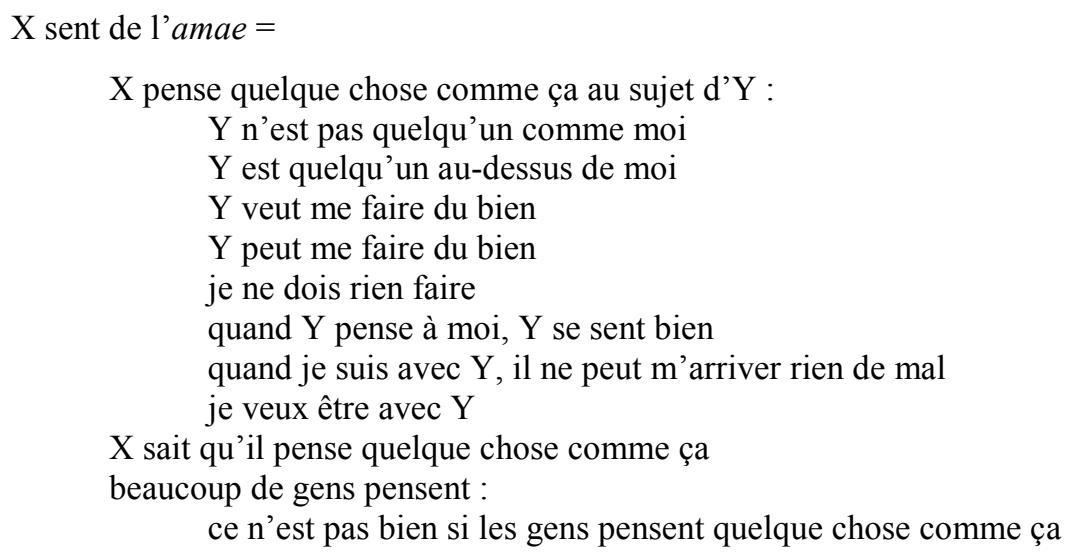

Dans cette formule sémantique n'ont été utilisés que des universaux : des structures grammaticales, des éléments lexicaux et des valences syntaxiques, auxquels s'ajoutent, au niveau typographique, des mises en retrait (dont le but est de grouper les composantes pour mieux montrer leur articulation au sein d'une formule sémantique). Les structures grammaticales, les éléments lexicaux (dits primitifs sémantiques, d'après l'anglais semantic primes) et les valences syntaxiques (du type PENSER QUELQUE CHOSE AU SUJET DE QUELQU'UN, où interviennent les primitifs PENSER, QUELQUE CHOSE et QUELQU'UN) sont universels, ou du moins présumés universels jusqu'à preuve du contraire, ce qui nous obligera peut-être à abandonner l'usage métaphorique (non spatial) du primitif AU-DESSUS (et des primitifs AU-DESSOUS, PRÈS et LOIN) dans la description des relations humaines (cf. Wierzbicka 1996 : 136, Wong 2006 : 121). Étant euxmêmes universels, le lexique et la grammaire de la métalangue confèrent à celle-ci un statut universel, culturellement neutre, et en font un outil descriptif dont la clarté et la rigueur restent inégalées.

\section{Un exemple franco-allemand : la Schadenfreude}

Pour illustrer à l'aide d'un autre exemple ce que permet d'accomplir la MSN, je reproduis ci-après quelques-unes des explicitations sémantiques proposées au cours des dernières années pour le mot Schadenfreude, mot allemand qui continue à briller par son absence de la plupart des dictionnaires français, mais qui se rencontre d'autant plus souvent dans le discours journalistique et aussi dans la presse spécialisée et de vulgarisation.

Pourquoi avoir choisi ce mot-là ? Tout simplement parce qu'il est évoqué dans ce qui est, sauf erreur, la seule communication au programme de sémantique de ce premier Congrès mondial de linguistique française à avoir eu recours à la MSN. Qu'il n'y en ait qu'une seule (à savoir celle d'Arkadiusz Koselak), sur les plusieurs dizaines qui ont été retenues, prouve que les tenants et aboutissants de la MSN sont encore peu connus des spécialistes de la langue française et des linguistes dont la langue de publication privilégiée est le français. Beaucoup d'entre eux n'apprécient pas encore ce qu'elle peut leur offrir ou bien la discréditent sur la base d'une familiarité superficielle. On pense en particulier à Patrick Sériot, auteur d'un article dans les Cahiers Ferdinand de Saussure (Sériot 2005) dont la lecture est déconseillée à ceux qui ne sont pas disposés à lire, en même temps, le texte que Wierzbicka a fait paraître en 2006 dans 
la même revue, à titre de droit de réponse. Ce texte est un condensé, enrichi de quelques paragraphes adressés à Sériot, d'une conférence faite au Collège de France en février $2004 .^{2}$

Koselak, lui, se situe aux antipodes de Sériot. Qui plus est, il n'en est pas à ses débuts. Il a d'ores et déjà publié plusieurs textes où la MSN fait l'objet d'une présentation destinée à un public francophone, ou bien est utilisée comme outil de description linguistique (Koselak 2003a/b, 2004, 2007a). Il est en outre l'auteur d'une thèse de doctorat (Koselak 2007b) où la MSN occupe une place très importante, thèse non encore publiée, mais dont on espère qu'elle le sera un jour, sous l'une ou l'autre forme, de sorte que la métalangue wierzbickienne puisse être portée à la connaissance du plus grand nombre. Il faut regretter que, dans sa communication au CMLF, Koselak ne se soit pas attardé aux principes de l'approche MSN. C'était sans doute inévitable, étant donné qu'il ne disposait que de quelques pages. Il se contente de renvoyer à un texte (Peeters 2002) qui, malheureusement, est d'ores et déjà relativement dépassé, de même qu'au site web (de langue anglaise) consacré à la MSN. On ajoutera que même les références bibliographiques plus récentes mentionnées à la fin de cette «prise de position » ne reflètent pas toujours l'état de la question, tant il est vrai que les mises au point et les révisions continuent - ce qui est signe d'un paradigme théorique vivant et vivace.

Étant donné que Koselak connaît assez bien les travaux de Wierzbicka, est-ce qu'on peut dire que l'usage qu'il fait de la MSN, du moins dans sa communication au présent congrès, emporte la conviction ? De la part d'un collègue qui s'intéresse de si près à l'entreprise wierzbickienne, on aurait pu s'attendre à une description de la notion de Schadenfreude qui ne laisse pas tant à désirer que la sienne. Commençons par un simple constat : l'explicitation qu'il propose, et qu'il dit avoir tirée de Wierzbicka (1999 : 104), est en fait très différente de ce qu'on trouve dans le soi-disant document source. Voici la version Koselak :

X pense :

beaucoup de bonnes choses sont arrivées à cette personne

cette personne a pensé : «c'est bon »

quelque chose de mauvais est arrivé à cette personne maintenant

je pense : c'est bon

à cause de cela $\mathrm{X}$ ressent quelque chose de bon

Cela ne correspond pas du tout à ce qu'on trouve dans Wierzbicka (1999) :

Schadenfreude (X felt Schadenfreude)

$\mathrm{X}$ felt something because $\mathrm{X}$ thought something

sometimes a person thinks about someone else :

« many good things happened to this person before now

this person thought : 'this is good'

something bad has happened to this person now

now I think : this is good»

when this person thinks this this person feels something good

$\mathrm{X}$ felt something like this

because $\mathrm{X}$ thought something like this

...et encore moins à ce qu'on trouve dans la préface de Wierzbicka à Peeters (2006), ouvrage auquel Koselak a eu accès, puisqu'il y renvoie dans sa thèse de doctorat. Je reproduis aussi bien la version anglaise que la version française, qui figurent dans ladite préface à côté d'une version allemande :

\section{$\mathrm{X}$ felt Schadenfreude $=$}

$\mathrm{X}$ thought like this about someone else :

« some good things happened to this person before

this person felt something good because of this

this person thought : this is good

now something bad happened to this person

this person feels something bad because of this 
I think : this is good»

when $\mathrm{X}$ thought like this $\mathrm{X}$ felt something good because of this

\section{$\mathrm{X}$ a ressenti de la Schadenfreude $=$}

$\mathrm{X}$ a pensé comme ça de quelqu'un d'autre :

« il y a des choses bien qui sont arrivées à cette personne avant cette personne a senti quelque chose de bien à cause de cela cette personne a pensé : cela est bien maintenant, quelque chose de mal est arrivé à cette personne cette personne sent quelque chose de mal à cause de cela je pense : cela est bien »

quand $\mathrm{X}$ a pensé comme ça, $\mathrm{X}$ a senti quelque chose de bien à cause de cela

On voit que les choix de Koselak ne sont pas toujours conformes à ceux qui ont été adoptés dans Peeters (2006) : il préfère BON à BIEN et MAUVAIS à MAL, alors qu'il y a de bonnes raisons d'opter pour des lexicalisations adverbiales plutôt qu'adjectivales (cf. Peeters $2006: 80-81$ ) ; et il opte pour RESSENTIR plutôt que SENTIR (sur le choix de ce dernier, voir Peeters 2006 : 101-102). BON, MAUVAIS et RESSENTIR sont encore utilisés dans Wierzbicka (2006a : 165). Dans l'état actuel de la recherche, PERSONNE, utilisé jusqu'il y a peu comme allolexe (c'est-à-dire variante) combinatoire de quelqu'un, alors qu'il semble être sémantiquement plus riche, n'est plus admis dans les explicitations. On adoptera donc comme explicitation du mot Schadenfreude dans une MSN française ni la version de Koselak, ni celle qui vient d'être citée, mais une troisième que voici :

\section{$\mathrm{X}$ a ressenti de la Schadenfreude $=$}

$\mathrm{X}$ a pensé comme ça au sujet de quelqu'un d'autre :

« des choses bien sont arrivées à ce quelqu'un avant

ce quelqu'un a senti quelque chose de bien à cause de cela

ce quelqu'un a pensé : cela est bien

maintenant, quelque chose de mal est arrivé à ce quelqu'un

ce quelqu'un sent quelque chose de mal à cause de cela je pense : cela est bien $»$

quand $\mathrm{X}$ a pensé comme ça, $\mathrm{X}$ a senti quelque chose de bien à cause de cela

Dans cette explicitation, comme dans celle de l'amae japonais ci-dessus, on trouve :

- un certain nombre des primitifs sémantiques qui relèvent de la version la plus récente du lexique de la MSN (voir en annexe un tableau, tiré de Peeters 2006, permettant de rapprocher entre elles les MSN de l'anglais, du français, de l'espagnol, du portugais et de l'italien)

- des prépositions qui ne font pas partie du lexique de la MSN, mais relèvent de valences syntaxiques universelles : PENSER [QUELQUE CHOSE] AU SUJET DE QUELQU'UN, QUELQU'UN D'AUTRE, ARRIVER À QUELQU'UN etc. (pour un aperçu de la grammaire universelle sur laquelle repose la MSN, voir par exemple Wierzbicka 2006c)

- des mises en retrait, dont le but est de regrouper des composantes qui vont ensemble (suite à une erreur de formattage, il n'y en a pas dans l'explicitation qui figure dans Wierzbicka 2006c : 198-199)

On y trouve en outre des passés composés, qui sont des allolexes (ou variantes) flexionnelles du présent ( $\mathrm{X}$ a pensé $=$ à un moment avant maintenant, $\mathrm{X}$ pense).

Comparons la définition ci-dessus à celles qu'on trouve sous la plume d'un certain nombre de journalistes francophones qui se sont occupés de Schadenfreude dans leurs écrits, et les avantages (mais aussi les désavantages !) de la MSN sauteront immédiatement aux yeux. Les extraits présentés ci-dessous ne constituent évidemment qu'un échantillon.

Face aux graves difficultés que rencontrent les Américains en Irak, les Français devraient éviter de tomber dans ce travers moral qu'on appelle en allemand 
«Schadenfreude», la joie honteuse consistant à se réjouir du malheur des autres. (Renaud Girard, Ne pas gêner les Américains, Le Figaro, 7 avril 2004)

Quand certains, outre-Atlantique ou outre-Manche, en sont à se demander publiquement si les Français n'ont pas eu raison depuis le début au sujet de l'Irak, il faut se garder de céder à la Schadenfreude, cette tentation de se réjouir du malheur des autres. (Patrick Sabatier, Retrait, Libération, 17 mai 2004)

Les Allemands appellent cela schadenfreude: prendre plaisir du malheur d'autrui. (Anne-Muriel Brouet, Les hommes aiment voir les «méchants » souffrir, La Tribune de Genève, 21 janvier 2006)

«Schadenfreude.» Pour résumer l'impact de l'affaire Hwang - la fraude sudcoréenne au clonage thérapeutique -, Evan Snyder, du Burnham Institute de la Jolla (Californie), use d'une expression allemande qui désigne la jubilation face au malheur d'autrui. «C'est décevant, nous ne disposons pas de cette technique, mais pour beaucoup d'équipes, cela signifie qu'elles sont encore dans le jeu », a résumé le chercheur (...). (Auteur inconnu, Débat aux Etats-Unis sur les cellules souches embryonnaires, Le Monde, 22 février 2006)

Face aux difficultés rencontrées par les Etats-Unis en Irak, beaucoup sont tentés de se laisser aller à ce que les Allemands appellent « Schadenfreude » : ils éprouvent du plaisir au malheur d'autrui. (Richard N. Haass, L'Irak est bien plus qu'un problème américain, Les Échos, 27 août 2007)

La langue allemande possède un mot très intéressant : schadenfreude. Ce mot décrit la compassion mais surtout le plaisir que beaucoup de gens ressentent face aux déboires des autres, surtout si ceux qui souffrent ont déjà été puissants et riches. Le procès de Conrad Black a fait ressortir beaucoup de schadenfreude. (Julius Grey, Le sort de Conrad Black, Journal de Montréal, 26 novembre 2007)

Il y a, dans ces définitions, beaucoup d'éléments que le locuteur français n'aura aucune difficulté à comprendre, dans la mesure où ils relèvent d'un univers culturel avec lequel il est familier. La plupart mentionnent les mots malheur des autres ou bien malheur d'autrui ; et ce malheur, qu'un seul d'entre eux appelle déboires des autres, provoque une réaction décrite à l'aide des verbes se réjouir, prendre plaisir, éprouver $d u$ plaisir, ressentir du plaisir et des noms jubilation et joie honteuse. Mais le mot Schadenfreude relève évidemment d'un autre univers culturel, proche mais en même temps assez différent; et la question doit se poser si les paraphrases proposées, dans la mesure où elles font intervenir des noms (malheur, déboires, jubilation, plaisir, joie), des verbes (se réjouir, éprouver, ressentir) et un adjectif (honteux) franco-français, font justice au mot qu'elles cherchent à élucider. Cinq journalistes sur six disent explicitement que c'est le terme allemand qu'ils expliquent, et non pas l'un ou l'autre usage français qui en est fait. Mais, comme dans le cas de l'amae japonais, ces explications sont des approximations. Pour aller au-delà d'une approximation, il faut recourir à ce qui est universel, car seul ce qui est universel est universellement intelligible et garantit que tout le monde attribue à un terme culturellement spécifique tel que Schadenfreude la même définition, à savoir la définition qu'y donnent... les Allemands. C'est là que la MSN présente un incomparable avantage : ses paraphrases sont longues, certes, mais elles sont immédiatement intelligibles, car formulées de sorte à être immédiatement, et sans risque de détournement sémantique, traduisibles dans toutes les langues du monde. Ce qui permet aux promoteurs de la MSN d'avancer cette thèse, que d'aucuns trouveront d'une arrogance inégalée, c'est le fait que la paraphrase fait intervenir des primitifs sémantiques, c'est-à-dire des universaux du lexique qui sont en outre maximalement simples du point de vue sémantique, et une grammaire qui, elle, est aussi universelle que les primitifs. Encore de l'arrogance ? Pas du tout : c'est grâce à plusieurs décennies d'enquêtes empiriques, sur des dizaines de langues typologiquement très différentes, que Wierzbicka et ses collaborateurs sont arrivés à concevoir un lexique et une grammaire qui peuvent à juste titre prétendre à l'universalité. On peut donc, à titre d'exemple, proposer, pour le mot schadenfreude, l'explicitation suivante dans une MSN malaise et une MSN coréenne, respectivement, tout en étant sûr que ce que comprendront les Malais et les Coréens est exactement la même chose que ce que comprennent les Français : ${ }^{3}$ 
(Malais) X rasa Schadenfreude $=$

$\mathrm{X}$ fikir tentang orang lain macam ini : « dulu banyak perkara (hal) yang baik terjadi kepada orang ini sebab itu, orang ini rasa sesuatu yang baik orang ini fikir : ini baik sekarang sesuatu yang buruk terjadi kepada orang ini sebab itu, orang ini rasa sesuatu yang buruk aku fikir : ini baik »

bila $\mathrm{X}$ fikir macam ini, sebab itu $\mathrm{X}$ rasa sesuatu yang baik

(Coréen) X 는 Schadenfreude 를 느꼈다 =

$\mathrm{X}$ 는 다른 어떤 사람에 대해 이렇게 생각했다: «이 사람에 게 전에 좋은 일들이 일어났다 이 사람은 이 때문에 무언가 좋은 느낌을 느꼈다

이 사람은 이는 좋다 라고 생각했다

지금 이사람에게 나쁜 일이 생겼다

이 사람은 이 때문에 나쁜 느낌을 느끼고 있다

나는 이는 좋다 라고 생각한다»

$\mathrm{X}$ 가 이렇게 생각할 때 $\mathrm{X}$ 는 이 때문에 무언가 좋은 느낌을 느낀다

\section{Pour conclure}

Parmi les spécialistes du français, la MSN ne connaît pas encore la renommée dont elle jouit ailleurs. Certes, beaucoup d'entre eux ont entendu parler de Wierzbicka, et peut-être de Cliff Goddard, depuis plusieurs années le bras droit de la linguiste polono-australienne. Mais rares sont ceux qui font l'effort de se tenir au courant ; la majorité renvoient à des textes, qu'ils ont lus ou non, et qui ont été publiés aux années quatre-vingts et quatre-vingt-dix, sans se soucier de ce qui a été accompli plus récemment. Et puis il y a tous ceux pour qui la notion d'une « métalangue sémantique naturelle » n'évoque absolument rien. C'est à eux, et à ceux qui ont besoin d'une mise à jour, que je me suis adressé dans ces quelques pages. ${ }^{4}$ Je voudrais que, dans leurs efforts de franchir le gué, ils continuent de sauter d'une pierre à une autre, sans jamais tomber à l'eau, mais aussi sans perdre l'autre rive de vue.

\section{Références bibliographiques}

Doi, T. (1982). Le jeu de l'indulgence : étude de psychologie fondée sur le concept japonais d'amae. Traduit du japonais par E. Dale Saunders. Paris : Éditions Le Sycomore / L'Asiathèque.

Goddard, C. (2002). Semantic primes and universal grammar in Malay (Bahasa Melayu). In C. Goddard \& A. Wierzbicka (dir.), Meaning and universal grammar: theory and empirical findings. Volume I. Amsterdam : John Benjamins, 87-172.

Goddard, C. ; Wierzbicka, A. (2002). Langue, culture et conceptualisation : la sémantique transculturelle. In N. Delbecque (dir.), Linguistique cognitive : comprendre comment fonctionne le langage. Bruxelles : De Boeck \& Larcie / Duculot, 161-188. Deuxième édition mise à jour (2006): 163-190.

Kleiber, G. (2001). Les référents évolutifs au large et à l'étroit. In W. De Mulder \& C. Schnedecker (dir.), Les référents évolutifs entre linguistique et philosophie. Metz : Université de Metz, 3-37.

Koselak, A. (2003a). La sémantique naturelle d'Anna Wierzbicka et les enjeux interculturels. Questions de communication, 4, 83-95.

Koselak, A. (2003b). Approche sémantique du concept de honte. Pratiques, 117-118, 51-76.

Koselak, A. (2004). Rêver : questions sémantiques. Le langage et l'homme, 39, 85-108. 
Koselak, A. (2007a). Sources et tradition polonaises en linguistique cognitive. Corela, Numéro spécial, Cognition, discours, contextes. Publié en ligne : http://edel.univ-poitiers.fr/corela/document.php?id=1626.

Koselak, A. (2007b). Sémantique des sentiments. "Quand je pense à toi, je ressens quelque chose de mauvais» en français et en polonais. Thèse de doctorat, Université Paul-Verlaine, Metz.

Peeters, B. (2002). La métalangue sémantique naturelle au service de l'étude du transculturel. Travaux de linguistique, 45, 83-101.

Peeters, B. (dir.) (2006). Semantic primes and universal grammar : empirical evidence from the Romance languages. Amsterdam : John Benjamins.

Peeters, B. (à paraître a). L'interculturel servi à la sauce MSN, ou À quoi sert la métalangue sémantique naturelle ?. Actes du Colloque international «Les enjeux de la communication interculturelle ». Montpellier : Maison des sciences de l'homme.

Peeters, B. (à paraître b). Les faux amis, une question de degré : l'apport de la métalangue sémantique naturelle. In M. Burston, F. Baider \& E. Lamprou (dir.), La marque en lexicographie: états présents, voies d'avenir. Limoges : Lambert-Lucas.

Sériot, P. (2005). Oxymore ou malentendu ? Le relativisme universaliste de la métalangue sémantique naturelle universelle d'Anna Wierzbicka. Cahiers Ferdinand de Saussure, 57, 23-43.

Wierzbicka, A. (1996). Semantics : primes and universals. Oxford : Oxford University Press.

Wierzbicka, A. (1999). Emotions across languages and cultures : diversity and universals. Cambridge : Cambridge University Press ; Paris : Maison des Sciences de l'homme.

Wierzbicka, A. (2006a). Sens et grammaire universelle : théorie et constats empiriques. Cahiers Ferdinand de Saussure, 59, 151-172. Version abrégée de Wierzbicka (2006c); texte traduit de l'anglais par Bert Peeters

Wierzbicka, A. (2006b). Les universaux empiriques du langage: tremplin pour l'étude d'autres universaux humains et outil dans l'exploration de différences transculturelles. Linx, 54, 151-179.

Wierzbicka, A. (2006c). Sens et grammaire universelle : théorie et constats empiriques. Linx, 54, 181-207. Version mise à jour d'une conférence faite au Collège de France en février 2004 ; texte traduit de l'anglais par Bert Peeters.

Wong, J. (2006). Social hierarchy in the «speech culture » of Singapore. In C. Goddard (dir.), Ethnopragmatics : understanding discourse in cultural context. Berlin : Mouton de Gruyter, 99-125.

Yoon, K-J. (2008). The Natural Semantic Metalanguage of Korean. In C. Goddard (dir.), Cross-linguistic semantics. Amsterdam : John Benjamins. 121-162.

\section{Annexe}

Versions anglaise, française, espagnole, portugaise et italienne du lexique de la MSN (d'après Peeters 2006 : 361-362 - à l'exception de IL Y A ... QUI, auquel on a substitué le plus ancien CERTAINS, puisque l'emploi de ce primitif ne semble pas devoir être limité à la position sujet)

\begin{tabular}{|c|c|c|c|c|}
\hline English & French & Spanish & Portuguese & Italian \\
\hline I & JE & YO & EU & IO \\
YOU & TU & TÚ & TU / VOCE & TU \\
SOMEONE & QUELQU'UN & ALGUIEN & ALGUÉM & QUALCUNO \\
SOMETHING & QUELQUE CHOSE & ALGO & ALGO & QUALCOSA \\
PEOPLE & GENS & GENTE & GENTE & GENTE \\
BODY & CORPS & CUERPO & CORPO & CORPO \\
\hline THIS & CE & ESTE & ESTE & QUESTO \\
SAME & MÊME & MISMO & MESMO & STESSO \\
OTHER & AUTRE & OTRO & OUTRO & ALTRO \\
\hline
\end{tabular}




\begin{tabular}{|c|c|c|c|c|}
\hline English & French & Spanish & Portuguese & Italian \\
\hline ONE & $\mathrm{UN}$ & UNO & $\mathrm{UM}$ & UNO \\
\hline TWO & DEUX & DOS & DOIS & DEUX \\
\hline SOME & CERTAINS & ALGUNOS & ALGUNS & C'̇̀ ... CHE \\
\hline MANY & BEAUCOUP & MUCHO & MUITO & MOLTO \\
\hline ALL & TOUT & TODO & TODO & TUTTO \\
\hline GOOD & BIEN & BUENO & $\mathrm{BOM}$ & BENE \\
\hline $\mathrm{BAD}$ & MAL & MALO & RUIM & MALE \\
\hline BIG & GRAND & GRANDE & GRANDE & GRANDE \\
\hline SMALL & PETIT & PEQUEÑO & PEQUENO & PICCOLO \\
\hline KNOW & SAVOIR & SABER & SABER & SAPERE \\
\hline THINK & PENSER & PENSAR & PENSAR & PENSARE \\
\hline WANT & VOULOIR & QUERER & QUERER & VOLERE \\
\hline FEEL & SENTIR & SENTIR & SENTIR & SENTIRE \\
\hline HEAR & ENTENDRE & OIR & OUVIR & SENTIRE \\
\hline SEE & VOIR & VER & VER & VEDERE \\
\hline SAY & DIRE & DECIR & DIZER & DIRE \\
\hline WORDS & MOTS & PALABRAS & PALAVRAS & PAROLE \\
\hline TRUE & VRAI & VERDAD & VERDADE & VERO \\
\hline DO & FAIRE & HACER & FAZER & FARE \\
\hline HAPPEN & ARRIVER & PASAR & ACONTECER & SUCCEDERE \\
\hline MOVE & BOUGER & MOVERSE & MEXER-SE & MUOVERSI \\
\hline THERE IS & IL Y A & HAY & TEM & C'È \\
\hline HAVE & AVOIR & TENER & TER & AVERE \\
\hline LIVE & VIVRE & VIVIR & VIVER & VIVERE \\
\hline DIE & MOURIR & MORIR & MORRER & MORIRE \\
\hline WHEN & QUAND & CUANDO & QUANDO & QUANDO \\
\hline NOW & MAINTENANT & AHORA & AGORA & ADESSO \\
\hline BEFORE & AVANT & ANTES & ANTES & PRIMA \\
\hline AFTER & APRES & DESPUÉS & DEPOIS & DOPO \\
\hline A LONG TIME & LONGTEMPS & MUCHO TIEMPO & MUITO TEMPO & MOLTO TEMPO \\
\hline A SHORT TIME & PEU DE TEMPS & POCO TIEMPO & POUCO TEMPO & POCO TEMPO \\
\hline FOR SOME TIME & POUR UN TEMPS & POR UN TIEMPO & POR ALGUM TEMPO & PER UN PO' DI TEMPO \\
\hline IN ONE MOMENT & EN UN MOMENT & EN UN MOMENTO & EM UM MOMENTO & IN UN MOMENTO \\
\hline WHERE & OU & DÓNDE & ONDE & DOVE \\
\hline HERE & ICI & AQUÍ & AQUI & QUI \\
\hline ABOVE & AU-DESSUS & ARRIBA & SOBRE & SOPRA \\
\hline BELOW & AU-DESSOUS & DEBAJO & DEBAIXO & SOTTO \\
\hline FAR FROM & LOIN & LEJOS & LONGE & LONTANO \\
\hline NEAR & PRES & CERCA & PERTO & VICINO \\
\hline SIDE & COTE & LADO & LADO & PARTE \\
\hline INSIDE & DANS & DENTRO & DENTRO & DENTRO \\
\hline TOUCH & TOUCHER & TOCAR & TOCAR & TOCCARE \\
\hline NOT & NE ... PAS & NO & NÃO & NON \\
\hline CAN & POUVOIR & PODER & PODER & POTERE \\
\hline MAYBE & PEUT-ETRE & TAL VEZ & TALVEZ & FORSE \\
\hline BECAUSE OF & A CAUSE DE & A CAUSA DE & POR CAUSA DE & A CAUSA DI \\
\hline IF & SI & SI & $\mathrm{SE}$ & SE \\
\hline VERY & TRÈS & MUY & MUITO & MOLTO \\
\hline MORE & PLUS & MÁS & MAIS & PIÙ \\
\hline
\end{tabular}




\begin{tabular}{|c|c|c|c|c|}
\hline \multirow{2}{*}{ English } & French & Spanish & Portuguese & Italian \\
\hline KIND OF & TYPE DE & TIPO DE & TIPO DE & TIPO DI \\
PART & PARTIE & PARTE & PARTE & PARTE \\
\hline LIKE & COMME & COMO & COMO & COME \\
\hline
\end{tabular}

1 D'après l'anglais semantic explication. Koselak (2003a: 88) s'est laissé induire en erreur en parlant des « explications verbales, comme préfère les appeler Anna Wierzbicka ». Le mot anglais explication est un dérivé du verbe explicate, qui ne doit pas se confondre avec le verbe explain. Ce dernier est à la base du nom explanation qui, lui, se traduit en français à l'aide du nom explication.

${ }^{2}$ Le texte complet, mis à jour, des deux conférences que Wierzbicka a prononcées au Collège de France en 2004 a été publié dans la revue Linx (Wierzbicka 2006b,c). On y trouvera une excellente introduction à l'approche MSN.

${ }^{3}$ Je remercie Cliff Goddard et Zifirdaus Adnan de m'avoir fourmi l'explicitation en malais, et Kyung-Joo Yoon d'en avoir fait autant pour l'explicitation coréenne. Sur la version malaise de la MSN, on verra Goddard (2002); sur la version coréenne, Yoon (2008).

${ }^{4}$ Un texte qui n'a pas été mentionné dans ce qui précède, et dont la lecture pourra également se révéler profitable, est celui que consacrent à la sémantique interculturelle Goddard \& Wierzbicka (2002). 